\title{
Desempenho ocupacional de cuidadores de crianças com transtorno do espectro autista (tea): uma revisão da literatura
}

Occupational performance of caregivers of children with autism spectrum disorder (asd): a literature review

Desempeño ocupacional de los cuidadores de niños con trastorno del espectro autista (tea): una revisón de lá literatura

Karina Saunders Montenegro ${ }^{1 *}$, Zelane Sousa dos Santos ${ }^{2}$, Ana Lidia Farias Bezerra², Jamilly Loyanne Silva do Rosário², Daniel Corrêa Coimbra².

\section{RESUMO}

Objetivo: Identificar as mudanças ocorridas no desempenho ocupacional de cuidadores de crianças com transtorno do espectro autista (TEA), e apresentar contribuições da Terapia Ocupacional para melhoria da qualidade de vida, diante das possíveis alterações. Métodos: Levantamento bibliográfico, de abordagem quanti-qualitativa, descritiva e exploratória da literatura brasileira afim de oportunizar informações sobre o desempenho ocupacional de cuidadores de crianças com TEA. Resultados: Identificou-se que ocorrem mudanças no desempenho ocupacional dos cuidadores, principalmente mudanças: físicas relacionadas a fadiga, dor, desconforto, sono, repouso e atividades físicas, psicólogicas, quanto a auto aceitação, capacidade de concentração, pensamentos e crenças pessoais e alterações na qualidade de vida. Para embasar a discussão desenvolveu-se três categorias de análise: Perfil dos cuidadores; Impacto do cuidar e o desempenho ocupacional do cuidador; Intervenção do terapeuta ocupacional. Considerações finais: os estudos identificaram que os cuidadores de crianças com TEA sofrem impactos no seu desempenho ocupacional, repercurtindo em seus aspectos físicos, psicológicos e sociais, podendo estes serem reduzidos através de intervenções e terapias direcionadas, dentre elas a terapia ocupacional.

Palavras-chave: Cuidadores, Desempenho ocupacional, Transtorno do espectro autista.

\begin{abstract}
Objective: Identify some changes which have been occurred in the occupational performance of Autistic Disorder Spectrum care children and to present the Occupational Therepy contribution to the life quality improvement, faced of possible alterations. Methods: Bibliographic apuration, a quanti qualitative approachment, explanatory and descriptive of brazilian literature in order tu opportunize informations about Autistic Disorder Spectrum care chinfrim occupational performance. Results: It has pointed there are some changes in caregiver occupational performance. Main changes: Physical related to fatigue, pain, discomfort, sleepy, rest, physical activities, psychologiatis, as self acceptance, concentration ability, thoughts, personal beliefs and life quality changes. To support the discussion, three categories of analysis were developed. Caregiver profile: Impact of caring and caregiver occupational performance, Occupational Therapist intervention. Final considerations: The researches have identified that the ADS care children suffer a impact on their occupational performance, reflecting in their physical aspects,social and psychological, can be reduced through interventions and directed therapies as Occupational Therapy.
\end{abstract}

Keywords: Caregiver, Occupational performance, Autistic spectrum disorder.

\footnotetext{
1 Universidade do Estado do Pará, Belém - PA. *E-mail: karinasmonte@yahoo.com.br

${ }^{2}$ Escola Superior da Amazônia, Belém - PA.
} 


\section{RESUMEN}

Objetivo: Identificar los cambios en el desempeño ocupacional de los cuidadores de niños con trastorno del espectro autista (TEA), además de presentar contribuciones de la terapia ocupacional para mejorar la calidad de vida, en vista de posibles cambios. Métodos: consiste en encuesta bibliográfica, con enfoque cuantitativocualitativo, descriptivo y exploratorio, con el fin de proporcionar información sobre el desempeño ocupacional de los cuidadores de niños con TEA. Resultados: Se señala que ocurren cambios en el desempeño ocupacional de los cuidadores, principalmente cambios fisicos relacionados con fatiga, dolor, molestias, sueños, descansos, actividades físicas y psicológicas con respecto a autoaceptación, la capacidad de concentración, pensamientos, creencias personales y cambios en la vida personal. A partir del análisis cualitativo, fue posible identificar las siguientes categorías: Perfil de los cuidadores; Cuidador em diferentes contextos de atención; Impacto del cuidador en la vida diaria de cuidado; El desempeño ocupacional del cuidador; Intervención del terapeuta ocupacional. Consideraciones finales: los estudios identificaron que cuidadores de niños com tea sufren impactos en su desempeño ocupacional, afectando aspectos físicos, psicológicos y sociales, que pueden reducirse mediante intervenciones y terapias específicas, como la terapia ocupacional.

Palabras clave: Cuidadores, Desempeño ocupacional, Trastorno del espectro autista.

\section{INTRODUÇÃO}

O Transtorno do Espectro Autista (TEA), classificado pelo Manual Diagnóstico e Estatístico de Transtornos Mentais (DSM-V) como um transtorno de neurodesenvolvimento, é marcado por comprometimentos nas habilidades de comunicação, interação social e de comportamento, apresentando níveis de comprometimento, classificados em leve, moderado e severo. Estes comprometimentos afetam tanto o indivíduo com TEA quanto aos seus cuidadores (APA, 2014; PINTO RNM, et al., 2016; CEZAR M, 2017).

De acordo com o tipo de deficiência e o grau de dependência, as tarefas irão variar e podem abalar a rotina de quem cuida. Ao se tornar um cuidador, o mesmo tende a descuidar-se da própria saúde, do seu bemestar físico, mental, emocional e social, isso ocorre por muitos motivos, dentre eles, a falta de tempo, a impossibilidade de deixar o paciente sozinho e o cansaço (BARROZO B, et al., 2015).

As mudanças no desempenho ocupacional de pais/cuidadores, principalmente nas atividades de vida diária (AVDs), se dão em virtude das necessidades de investirem tempo nos cuidados das crianças com TEA, postergando os cuidados consigo (FILHA FSSC, et al., 2018). Por esta razão, doenças surgem e diversos impactos sociais como: a depressão, tensão, desgastes nos relacionamentos afetivos, perda de perspectiva de vida, mudanças na rotina, interrupções nas tarefas do cotidiano, problemas financeiros, entre outros (SANTOS AL e SANTOS JLF, 2015).

Diante deste cenário a Terapia Ocupacional contribui no processo de reorganização da rotina e melhoria da qualidade de vida deste cuidador, com estratégias que impactam diretamente em seus contextos e ambientes, promovendo redução do nível de estresse e melhor desempenho ocupacional (ESTANIESKI II e GUARANY NR, 2015). A Terapia Ocupacional identifica a relevância da relação mente-corpo-espírito, com o intuito de compreender e intervir com o cuidador, a partir do trabalho da sua vida e das relações que estabelece de cuidado. As ações da terapia ocupacional visam propiciar comodidade no desempenho ocupacional dos cuidadores, de forma que cooperem para a organização do seu cotidiano (AOTA, 2015).

Desta forma, esta pesquisa objetiva conhecer as alterações na performance de desempenho ocupacional de cuidadores de crianças com TEA, tendo como base artigos já publicados em revistas cientificas brasileiras e assim responder as seguintes perguntas norteadoras: os cuidadores de crianças com TEA apresentam alterações de desempenho ocupacional em seu cotidiano? Diante das alterações quais as possíveis estratégias de intervenção da terapia ocupacional para a promoção da qualidade de vida desses cuidadores? 


\section{MÉTODOS}

Revisão Integrativa da literatura brasileira, de abordagem quantitativa descritiva e exploratória. Foram selecionados artigos científicos encontrados no acervo bibliográfico da Biblioteca Virtual de Saúde (BVS) e nas bases de dados: Literatura Latino-Americana e do Caribe em Ciências da Saúde (Lilacs), Scientific Electronic Library Online (Scielo), Medical Literature Analysis and Retrieval System Online (Medline), Caderno Brasileiro de Terapia Ocupacional da Universidade Federal de São Carlos (UFSCAR) e Revista de Terapia Ocupacional da Universidade de São Paulo (USP). Para a busca foram utilizados como descritores: cuidadores, desempenho ocupacional, transtorno do Espectro Autista. Os descritores foram utilizados separados e de forma associada.

Foram critérios de inclusão: estudos com textos completos e disponíveis de maneira gratuita, textos em português, estudos com cuidadores de crianças e estudos realizados no período de 2009 e 2019 . O levantamento ocorreu nos meses de setembro a outubro de 2019. Foram critérios exclusão: textos incompletos referentes ao tema, textos em outros idiomas, estudos que não foram publicados no período de 2009 e 2019 , carta ao editor, anais de eventos e descrição de congressos, simpósios e palestras. Após os critérios o material selecionado foi analisado a partir da leitura prévia dos resumos, após análise foram excluídos os estudos cujo foco não evidenciou relação com o tema.

Figura 1 - Fluxograma para seleção dos estudos.

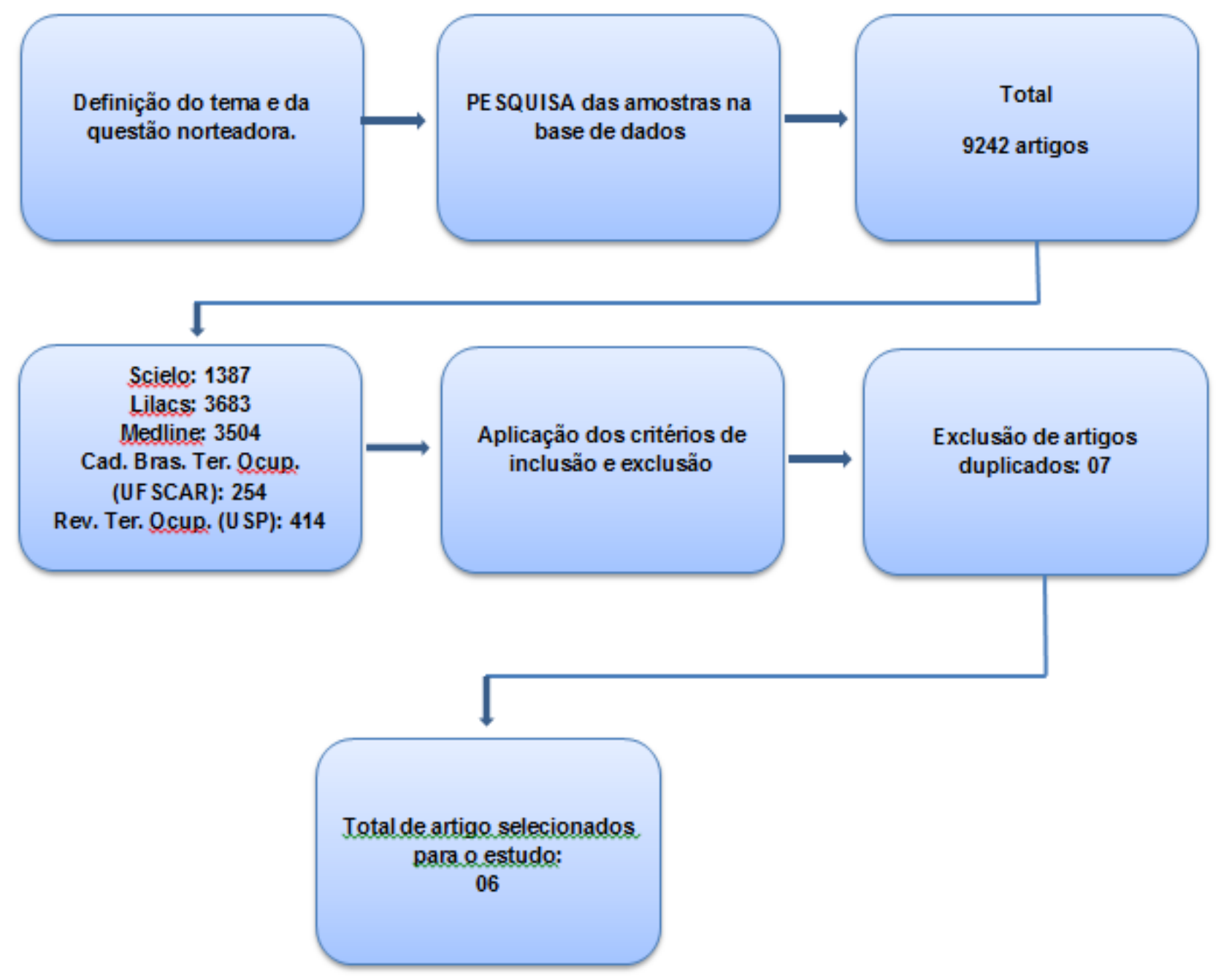

Fonte: Montenegro KS, et al., 2020. 
Os dados quantitativos foram registrados em frequência e porcentagem correspondendo ao: ano de publicação, região do local de publicação, autores (profissões e vínculos com universidades), caracterização de estudo. Estes dados estão apresentados em gráficos e/ou tabelas que ajudaram na identificação do perfil dos trabalhos produzidos com esta temática no Brasil nos últimos dez (10) anos.

Para facilitar a discussão das perguntas norteadoras os dados foram organizados em três categorias de análise: Perfil dos cuidadores; Impacto do cuidar e o desempenho ocupacional do cuidador; Intervenção do terapeuta ocupacional.

\section{RESULTADOS E DISCUSSÃO}

Serão apresentados os dados quantitativos referentes ao ano de publicação, local de publicação, características dos autores de publicação, caracterização dos estudos e perfil dos estudos da pesquisa. Durante o levantamento de dados foram encontrados um total de 9.242 artigos (Tabela 1).

Tabela 1 - Estudos localizados na busca.

\begin{tabular}{cccccc}
\hline Descritores/combinações & Scielo & Lilacs & Medline & $\begin{array}{c}\text { Card. De to. } \\
\text { (ufscar) }\end{array}$ & $\begin{array}{c}\text { Rev. De } \\
\text { to (usp) }\end{array}$ \\
\hline Cuidadores & 1.387 & 3.005 & 2846 & 20 & 88 \\
Desempenho ocupacional & 0 & 350 & 497 & 80 & 256 \\
Tea + cuidadores & 0 & 285 & 139 & 31 & 7 \\
$\begin{array}{c}\text { Desempenho ocupacional } \\
\text { + cuidadores }\end{array}$ & 0 & 43 & 22 & 123 & 63 \\
\hline
\end{tabular}

Fonte: Montenegro KS, et al., 2020.

Foram selecionados apenas o material que atendia aos critérios de inclusão e exclusão definidos neste estudo. Foram excluídos 7 artigos, pois os mesmos se repetiam em outras bases de dados. Assim foram utilizados neste estudo 6 artigos (Tabela 2).

Tabela 2 - Estudos selecionados após aplicação dos critérios de exclusão e inclusão.

\begin{tabular}{cccccc}
\hline Descritores/combinações & Scielo & Lilacs & Medline & Card. De to (ufscar) & Rev. De to (usp) \\
\hline Cuidadores & 2 & 0 & 0 & 0 & 0 \\
Desempenho ocupacional & 0 & 0 & 0 & 01 & 0 \\
Tea + cuidadores & 0 & 02 & 0 & 0 & 01 \\
Desempenho ocupacional & 0 & 0 & 0 & 0 & 0 \\
+ cuidadores & & & & & $\mathbf{0 6}$ \\
\hline Total no de artigos & & &
\end{tabular}

Fonte: Montenegro KS, et al., 2020.

Através da pesquisa identificou-se que a maioria dos trabalhos selecionados ocorreu no ano de 2015 (50\%), os demais artigos foram publicados em 2014 (16,6\%) e em $2016(33,3 \%)$, não havendo nenhuma publicação referente ao tema entre 2009 à 2013 e após o ano de 2016. Estima-se que 1\% das crianças e adolescentes do mundo estão na condição do transtorno do espectro autista, dados retratam o aumento de casos de TEA nos últimos anos, e consequentemente o aumento de cuidadores (SILVA CM, 2015; TEIXEIRA G, 2017).

Desta forma, esperava-se também um aumento nas pesquisas sobre o tema ao longo do tempo. Pois através das pesquisas que são analisadas e identificadas as principais dificuldades em uma determinada área, e se tratando de um assunto tão importante, os estudos são fundamentais para apontar as principais alterações no cotidiano desses cuidadores.

O desempenho ocupacional e a qualidade de vida dos cuidadores de crianças com TEA são afetados, assim, as atividades ocupacionais não são desempenhadas de maneira adequada, pois acabam sendo trocadas ou excluídas da rotina, por haver uma sobrecarga persistente e uma maior responsabilidade no cuidado da criança com TEA. As principais atividades afetadas são as atividades do autocuidado e lazer. 
Contudo, o desenvolvimento de pesquisas nesta área torna-se fundamental para que os profissionais saíbam as condutas necessárias para lidar com as dificuldades sofridas pelo cuidador (ESTANIESKI II e GUARANY NR, 2015).

Quanto ao local de publicação observa-se que a maioria dos artigos selecionados para o estudo foram publicados na região Sudeste, com o quantitativo de 50\% no estado de São Paulo, 33,32\% na região Sul, nos estados de Rio Grande do Sul e Paraná, ambos com 16,66\% e na região Nordeste com quantitativo de 16,66\% no estado de Sergipe. Não foram encontrados artigos produzidos na região Norte (Tabela 3).

Tabela 3 - Local de Publicação.

\begin{tabular}{ccc}
\hline Publicado & Quantidade & Porcentagem \\
\hline Rio Grande do Sul & 1 & 16,66 \\
São Paulo & 3 & 50 \\
Paraná & 1 & 16,66 \\
Sergipe & 1 & 16,66 \\
\hline
\end{tabular}

Fonte: Montenegro KS, et al., 2020.

Nota-se o maior número de publicações na região Sudeste, um dado significativo levando em consideração a magnitude do nosso país. Deve-se levar em consideração que existe uma variação significativa a nível cultural, regional e social que impactam diretamente na relação de cuidado. Assim, também seria importante o desenvolvimento de mais estudos, em diferentes contextos, regiões e culturas.

Identificou-se que $83 \%$ dos autores são profissionais da saúde e apenas $5,5 \%$ da educação, $11,1 \%$ ciências humanas. Todos os autores são de universidade pública, salientando a importância da equipe multiprofissional na assistência à criança com TEA e seu respectivo cuidador (Tabela 4).

Tabela 4 - Características dos Autores.

\begin{tabular}{ccc}
\hline Profissões & Quantidade & Porcentagem \\
\hline Acadêmica de Letras & 1 & 5,5 \\
Enfermeira & 2 & 11,1 \\
Fonoaudiólogo & 3 & 16,6 \\
Neuropsicólogo & 2 & 11,1 \\
Odontólogo & 4 & 22,2 \\
Psicólogo & 2 & 11,1 \\
Terapeuta Ocupacional & 4 & 22,2 \\
\hline
\end{tabular}

Fonte: Montenegro KS, et al., 2020.

Estima-se que no Brasil existam cerca de 200 mil cuidadores que necessitam esclarecer suas dúvidas e expor suas angústias, pois, dessa forma, é possível a elaboração de ações que o orientem melhor nos cuidados com o paciente. É também fundamental o acompanhamento multidiciplinar para com o cuidador. É urgente a necessidade de se estruturar um grupo multidisciplinar qualificado, com amplo conhecimento sobre o TEA, na busca da qualidade de vida da criança e de seu cuidador (SOUZA JM e MILOR V, 2015).

Quanto ao método utilizado nos estudos observou-se que a maioria utilizou abordagem quantitativa (50\%) seguida da abordagem mista, quanti-qualitativa com 33,3\% (Tabela 5).

Tabela 5 - Caracterização de Estudos.

\begin{tabular}{ccc}
\hline Tipo de abordagem & Quantidade & Porcentagem \\
\hline Qualitativa & 1 & 16,6 \\
Quantitativa & 3 & 50 \\
Quanti-qualitativa & 2 & 33,3 \\
\hline
\end{tabular}

Fonte: Montenegro KS, et al., 2020.

Em relação ao tipo de estudo evidenciou-se que todos são pesquisas de campo e que na maioria dos estudos pesquisados, os pesquisadores tiveram um contato direto com a clientela em estudo, o que enfatiza que durante a realização de uma pesquisa de campo algumas questões precisam ser colocadas de forma 
imediata, pois a necessidade de dar conta dessas questões para poder encerrar as etapas da pesquisa, levando em consideração as reflexões em torno dos problemas enfrentados, erros cometidos, escolhas feitas e dificuldades encontradas. A partir desta perspectiva, é fundamental o estudo de campo, pois é desta maneira que o pesquisador fica frente a frente com a realidade das instituições, podendo identificar as maiores dificuldades apresentadas.

Ao descrever os artigos, observa-se que no artigo A.1, o objetivo do estudo foi avaliar a sobrecarga de familiares cuidadores de crianças com Transtornos do Espectro Autista, segundo a percepção dos próprios cuidadores. Os resultados demonstraram que os grupos estavam moderadamente sobrecarregados (MISQUIATTI ARN, 2015).

O artigo A.2 objetivou identificar, sob a ótica das famílias de crianças e adolescentes com TEA, as experiências cotidianas e suas demandas na realidade de cuidados dispensados aos filhos em três fases do desenvolvimento. Foram identificadas dificuldades comuns aos participantes nas atividades sociais, na comunicação, na dependência nas atividades de autocuidado e higiene (MINATEL MM e MATSUKURA TS, 2014).

O artigo A.3 teve como objetivo identificar a relação do padrão de independência da criança com TEA e o nível de auto eficácia do seu cuidador. O estudo apontou para a ausência de correlação do nível de dependência da criança com TEA e da percepção de auto eficácia do cuidador, sugerindo limitação e particularidade da amostra, cujos cuidadores se manifestaram com disponibilidade prática e afetiva, para atender às demandas da criança (TABAQUIM MLM, et al.,2015).

O artigo A.4 analisou o desempenho ocupacional, qualidade de vida e o estresse de mães que eram cuidadoras de crianças e adolescentes com TEA. A pesquisa em questão demonstrou que existe uma correlação direta entre ser cuidadora de uma criança e ou adolescente com TEA e apresentar alterações no desempenho ocupacional, qualidade de vida e estresse, apontou também que as mães/cuidadoras apresentam uma baixa qualidade de vida, alterações no desempenho ocupacional e altos níveis de estresse. (ESTANIESKI II e GUARANY NR, 2015).

O artigo A.5, objetivou avaliar o perfil e a sobrecarga de cuidadores de crianças/adolescentes. As avaliações foram realizadas por meio dos questionários sobre o perfil e a sobrecarga dos cuidadores e o perfil das crianças/adolescentes. Estes questionários foram aplicados a 168 cuidadores.

As crianças/adolescents necessitavam significativamente de maior auxílio para a alimentação, banho, vestuário, controle de esfíncteres e higiene íntima. Os cuidadores apresentaram sobrecarga moderada, quando comparados à cuidadores de crianças/adolescentes típicas (BARROS ALO, et al., 2017).

E por fim, o artigo A.6 teve como objetivo identificar a sobrecarga em 82 cuidadores/familiares de crianças e adolescentes atendidos no Centro de Atenção Psicossocial infantil do município de Maringá, Paraná, Brasil. Foi identificado sobrecarga elevada quanto à assistência em atividades da vida cotidiana, como supervisão na tomada de medicamentos e sobrecarga subjetiva alta decorrente do convívio com comportamentos problemáticos (BURIOLA AA, et al., 2016). Nesse contexto é imprescindível vincular a família nas ações de cuidado prestadas nos serviços de saúde, sendo necessário que os profissionais atuem potencializando pontos positivos da convivência da família com essas crianças e adolescentes, sanando dúvidas e sendo o suporte da família para as dificuldades que surgem no cotidiano do cuidado.

Quanto ao perfil dos artigos encontrados observou-se que os perfis dos artigos consistem em maior prevalência de artigos publicados no ano de 2015 (50\%), com destaque para a região Sudeste do Brasil $(50 \%)$, sendo a maioria dos autores profissionais da saúde (83\%), destes $22,2 \%$ são Terapeutas Ocupacionais. Todos os artigos encontrados foram de pesquisa de campo.

Quanto aos dados qualitativos desenvolveu-se a leitura exaustiva dos artigos. Assim, foi possível responder as perguntas norteadoras desse estudo, no qual constata-se que de acordo com os artigos os 
cuidadores de crianças diagnosticadas com transtorno do espectro autista (TEA) apresentam alterações de desempenho ocupacional em seu cotidiano a partir do seu novo papel de cuidador. Apresentando alterações em seu desempenho ocupacional, AVD, AIVD, participação social, trabalho e lazer.

Diante das alterações ocorridas o terapeuta ocupacional pode desenvolver estratégias de organização da rotina, promover a participação do cuidador em ocupações positivas que promovam a saúde, podendo melhor avaliar como cada aspecto contribui para as preocupações relacionadas com desempenho dos cuidadores, iminentemente contribuindo para intervenções que irá auxiliar no desempenho ocupacional.

\section{Perfil dos cuidadores}

Todos os artigos relataram que o papel de cuidador é na maioria das vezes realizado por mulheres. No núcleo familiar as mães são particularmente vulneráveis, uma vez que assumem o papel de cuidador principal na maioria das famílias. Apresentam sobrecarga emocional, física e financeira, além de restrições nas atividades sociais e de lazer (BOURKE TH, et al., 2011; BAGHDADLI A, et al., 2014). A figura feminina como cuidadora enfatiza a tradição histórica e cultural da mulher em assumir a responsabilidade principal do ato de cuidar, indicando que independe do tipo de condição da pessoa que recebe cuidados e do grau de parentesco do cuidador. Quando há necessidade de cuidados, existe uma pressão social e familiar para que elas os prestem.

Conforme descrito no artigo A.3, a relação de gênero, distribuído aos cuidadores, indica que o modelo da família convencional brasileira, no qual o cuidar dos filhos especialmente aqueles portadores de cuidados especiais são representados pela mãe (Vieira AJM, 2016). Com isso essa divisão sexual entre os cuidadores parece amparada na vivência da maternidade, determinando assim que as mulheres estariam designadas para lidar com as atividades de cuidado, ensinada às mulheres dentro da própria família, através das gerações (WEGNER W e PEDRO ENR, 2010).

Quanto pior o desempenho ocupacional e satisfação na realização das atividades, pior será a qualidade de vida nas mães, principalmente mães de criaças com TEA. Assim, uma vez que assumem o papel principal de cuidadora tendem a ter a maior responsabilidade nos cuidados prestados, ficando mais vulneráveis a sobrecargas. A rotina exaustiva afeta sua saúde física e mental, por vezes abrem mão das suas ocupações, para dar melhor suporte ao cuidado, afetando seu desempenho ocupacional. (ESTANIESKI II e GUARANY NR, 2015).

\section{Impacto do cuidar e o desempenho ocupacional do cuidador}

Todos os artigos retrataram que o cuidar prejudica a saúde física e mental, vida social, familiar e financeira do cuidador, impactando direto e indiretamente em sua qualidade de vida, fazendo com que o cuidador fique mais vulnerável a doenças, gerando impacto negativo tanto para o cuidador quanto para a pessoa cuidada. O artigo A.6 conclui que a doença mental tem vários impactos na vida dos familiares de uma criança que necessita de cuidados especiais, um deles é o fato da família se sobrecarregar e dispor de suas necessidades e desejos colocando-os em segundo plano (BURIOLA AA, et al., 2016).

Desta forma, nota-se que o impacto resulta em um adoecimento ao lindar com a incapacidade e dependência do indivíduo cuidado, ocasionando em um grande abalo social. A maioria dos artigos enfatizam que o cuidar interfere diretamente na vida ocupacional do cuidador, devido desempenhar esse papel suas ocupações são interrompidas, transferindo ou excluindo da sua rotina em razões de sobrecarga física ou mental, deixando de investir tempo para realizar suas próprias ocupações (ESTANIESKI II e GUARANY NR, 2015; PINTO RNM, et al., 2016).

Barrozo B, et al. (2015) consideram que as mudanças que ocorrem no desempenho ocupacional do cuidador, se refletem principalmente nas atividades de vida diária (AVD's), atividades instrumentais de vida diária (AIVD's), tais como participação social, lazer, descanso e sono, e a principal alteração refere-se a falta de tempo em realizá-las. 


\section{Intervenção do Terapeuta Ocupacional}

Os artigos A.2 e A.4 mostram o papel do terapeuta ocupacional na vida do cuidador, que contribui no processo de promoção de vida e saúde do mesmo, ajudando na reorganização de suas ocupações que foram debilitadas, promovendo o bem-estar físico e emocional. $O$ artigo A.2 ressalta ainda que, as contribuições da terapia ocupacional são de extrema relevância, pois este profissional irá atuar na busca da produtividade, favorecendo ações construídas no cotidiano de acordo com o contexto social vivido por cada indivíduo, priorizando sua realidade e singularidade (MINATEL MM e MATSUKURA TS, 2014).

Dessa forma, evidencia-se que as mudanças que ocorrem no desempenho ocupacional do cuidador, compromete seu próprio auto cuidado e seu bem-estar físico e emocional. Contudo, a terapia ocupacional apresenta soluções para que esse cuidador obtenha uma melhor qualidade de vida, abrangendo em suas ocupações onde tem dificuldades em desenvolvê-las minimizado no impacto do cuidar.

Em particular, destaca-se a importância de apoiar as mães no desenvolvimento de uma rotina estruturada, com previsibilidade e com a participação de todos os membros da família, para que assim resulte na organização e harmonia da unidade familiar (RODGER S e UMAIBALAN V, 2011).

Para afirmar a credibilidade dos serviços centrados na família, Lee LC, et al. (2008) identificaram que a diminuição do estresse dos pais garante melhores/ positivos resultados no desenvolvimento da criança e, o estabelecimento de intervenções básicas e auxílios para as necessidades das famílias contribuem no planejamento do futuro familiar.

As intervenções da terapia ocupacional dedicam-se a criar oportunidades para que o indivíduo se envolva em ocupações, o conduzindo a participar de acontecimentos que tragam significado para sua vida. $O$ terapeuta ocupacional desenvolverá em comum acordo com o cliente propiciando metas ao indivíduo com o objetivo de melhorar a saúde, reconstruindo suas ocupações, dando - Ihe melhor qualidade de vida, reduzindo os níveis de estresse, adequando seu desempenho ocupacional (AOTA, 2015; ESTANIESKI II e GUARANY NR, 2015).

\section{CONSIDERAÇÕES FINAIS}

O cuidador de crianças com TEA é na maioria das vezes a própria mãe, que vive em função das necessidades da criança, afetando assim o seu desempenho ocupacional, gerando impactos físicos, psicológicos e sociais como a depressão, tensão, desgastes nos relacionamentos afetivos, perda de perspectiva de vida, mudanças na rotina, dificuldades financeiras, bem como a falta de tempo para realizar suas atividades de vida diária e laborais, dedicando-se integralmente na maioria das vezes ao cuidado da criança. Essas repercussões na vida do cuidador podem ser reduzidos através de intervenções e terapias direcionadas, dentre elas a terapia ocupacional. As intervenções terapêuticas ocupacionais contribuem para estabelecer rotinas estruturadas, com foco na reorganização da dinâmica familiar, realiza treinamentos, estratégias facilitadoras de cuidado e desenvolve adaptações para facilitar a rotina, o cotidiano, oferecendo apoio as necessidades do cuidador e contribuindo para melhora de sua qualidade de vida.

\section{REFERÊNCIAS}

1. AMERICAN OCCUPATIONAL THERAPY ASSOCIATION (AOTA). Estrutura da prática da Terapia Ocupacional: domínio \& processo - 3 ${ }^{\underline{a}}$ ed. traduzida. Revista De Terapia Ocupacional Da Universidade De São Paulo, 2015; 26(esp), 1-49.

2. AMERICAN PSYCHIATRIC ASSOCIATION (APA). Transtornos mentais. DSM - V. In: Manual diagnóstico e estatístico de transtornos mentais. 5nd ed. Porto Alegre: Artmed, 2014; 50p.

3. BAGHDADLI A, et al. Impacto do autismo em adolescentes na qualidade de vida dos pais. Qual Life Res, 2014; 23(16): 1859-1868.

4. BARROS ALO, et al. Sobrecarga dos cuidadores de crianças e adolescentes com Síndrome de Down. Ciência \& Saúde Coletiva, 2017; 22(11): 3625-3634. 
5. BARROZO B, et al. As alterações nos papéis ocupacionais de cuidadores de pessoas com deficiência visual. Ver. Ter. Ocup. Univ. São Paulo, 2015; 26(3): 409-417.

6. BOURKE TH, et al. Barreiras à participação da força de trabalho material e relação entre trabalho remunerado e saúde. J Intellect Disabil Res, 2011; 55(5): 511-520.

7. BURIOLA AA, et al. Sobrecarga dos cuidadores de crianças ou adolescentes que sofrem transtorno mental no município de Maringá - Paraná. Escola Anna Nery, 2016; 20(2) Abr-Jun.

8. CEZAR M. Autismo afeta cerca de 1\% da população. Ciências \& amp; Cognição, 2017; 22(1): 041-062. Disponível em: http://www.saude.mg.gov.br/ajuda/story/6884-autismo-afeta-cerca-de-1-da-populacao. Acesso em: 13 abr. 2020.

9. ESTANIESKI II, GUARANY NR. Qualidade de vida, estresse e desempenho ocupacional de mães cuidadoras de crianças e adolescentes autistas. Rev. Ter. Ocup. Univ. São Paulo. 2015; 26(2): 194-200.

10. FILHA FSSC, et al. Coping e estresse familiar e enfrentamento na Perspectiva do transtorno do espectro do autismo. Rev. Cient. Sena Aires. 2018; 7(1): 23-30.

11. LEE LC, et al. Crianças com autismo: qualidade de vida e preocupação dos pais. J Autism Dev Disord, 2008; 38: 1147-60.

12. MINATEL MM e MATSUKURA TS. Famílias de crianças e adolescentes com autismo. Ver. Ter. Ocup. Univ. São Paulo, 2014; 25(2):126-34.

13. MISQUIATTI ARN, et al. Sobrecarga familiar e crianças com transtornos do espectro do autismo: perspectiva dos cuidadores. Rev. CEFAC, 2015; 17(1): 192-200.

14. PINTO RNM, et al. Autismo infantil: impacto do diagnóstico e repercussões nas relações familiares. Rev. Gaúcha Enferm, 2016; 37(3): e61572.

15. RODGER S e UMAIBALAN V. As rotinas e rituais de famílias de crianças em desenvolvimento típico em comparação com famílias de crianças com transtorno do espectro do autismo: um estudo exploratório. British Journal of Occupational Therapy, 2011; 74(1): 20-26.

16. SANTOS AL e SANTOS JLF. O desempenho de papéis ocupacionais de idosos sem e com sintomas depressivos em acompanhamento geriátrico. Rev. Bras. Geriatr. Gerontol. Rio de Janeiro, 2015; 18(2): 273-283.

17. SILVA CM. Autismo e vitamina D: uma revisão da literatura. Trabalho de conclusão de curso (Bacharel Nutrição) Faculdade de Ciências da Saúde da Universidade de Brasília - UnB, Brasilia, 2015; 26p.

18. SOUZA JM e MIOR V. Desenvolvimento infantil: análise de um novo conceito. Rev. Latino-Am. Enfermagem nov.dez, 2015; 23(6): 1097-104.

19. TABAQUIM MLM, et al. Autoeficácia de cuidadores de crianças com o transtorno do espectro autista. Rev. Psicopedagogia, 2015; 32(99): 285-92.

20. TEIXEIRA, G. Manual do Autismo: guia dos pais para o tratamento completo. 3nd ed. Rio de Janeiro: BestSeller, 2017; 93p.

21. VIEIRA AJM. Impactos de uma criança autista na família e o papel da terapia ocupacional neste ambiente. Trabalho de conclusão de curso (Bacharel em terapia ocupacional) - Universidade Federal de Minas Gerais, Belo Horizonte, 2016; 27p.

22. WEGNER W e PEDRO ENR. Os múltiplos papéis sociais de mulheres cuidadoras-leigas de crianças hospitalizadas. Ver. Gaucha. Enferm, 2010; 31(2): 335-342. 